\title{
Padrões de Uso de Drogas e Problemas Associados em Adolescentes Judicializados
}

\author{
André Vilela Komatsu' \\ Elvio Luciano Bono² \\ Marina Rezende Bazon ${ }^{1}$ \\ ${ }^{1}$ Universidade São Paulo, Ribeirão Preto, São Paulo, Brasil \\ ${ }^{2}$ Centro Universitário UNIFAFIBE, Bebedouro, São Paulo, Brasil
}

\begin{abstract}
Resumo
Objetivou-se caracterizar uma amostra de adolescentes em conflito com a lei em relação ao consumo de substâncias e possíveis problemas associados. Participaram 120 adolescentes do sexo masculino, entre 13 e 18 anos, custodiados na Unidade de Atendimento Inicial da Fundação CASA, que responderam ao Drug Use Screening Inventory - Revised (DUSI-R). Análises descritivas indicaram que a maioria dos adolescentes teria feito uso de maconha (84\%) e álcool (54\%). Em relação ao padrão de consumo, $52 \%$ foram categorizados em uso abusivo ou de provável dependência. Quanto aos problemas associados ao consumo, os participantes possuem densidade média superior aos da população normativa nos dez domínios investigados pelo DUSI-R, com destaque aos Transtornos $(M=42)$, Escola $(M=50)$ e Pares $(M=55)$. Vale observar que 37\% da amostra se declararam abstêmios, e que os desvios das densidades de problemas sugerem alta heterogeneidade nos níveis de necessidades/dificuldades, o que implica ultrapassar discursos que propalam a uniformidade dessa população.

Palavras-chave: álcool, adolescentes em conflito com a lei, delinquência juvenil, uso de substâncias
\end{abstract}

\section{Drug Use Patterns and Associated Problems Among Adolescent Offenders}

\begin{abstract}
This study aimed to characterize a sample of adolescents in conflict with the law regarding substance use and potential associated problems. The sample consisted of 120 male adolescents aged 13 to 18 in custody at the Initial Care Unit from Fundação CASA (Center for Adolescent Socio-Educational Care) in the state of São Paulo, who responded to the Drug Use Screening Inventory - Revised (DUSI-R). Descriptive analyses indicated that most adolescents were reported to have used marijuana $(84 \%)$ and alcohol (54\%). Regarding the pattern of consumption, $52 \%$ were categorized as abusive use or probable dependence. Regarding the problems associated with consumption, participants showed a higher mean density than the normative population in the ten domains investigated by the DUSI-R, with emphasis on Disorders $(M=42)$, School $(M=50)$, and Peers $(M=$ 55). It is worth noting that $37 \%$ of the sample declared themselves abstainers and that the deviations of the problem densities suggest high heterogeneity in the levels of needs/difficulties, which implies overcoming discourses that promote the uniformity of this population.
\end{abstract}

Keywords: alcohol; adolescents in conflict with the law; juvenile delinquency; substance use

\section{Patrones de Consumo de Drogas y Problemas Asociados en Adolescentes Infractores}

\begin{abstract}
Resumen
Este estudio tuvo como objetivo caracterizar una muestra de adolescentes en conflicto con la ley por el consumo de sustancias y posibles problemas asociados. Participaron de la muestra 120 adolescentes varones de 13 a 18 años en custodia de la Unidad de Atención Inicial de la Fundación CASA, quienes respondieron al Drug Use Screening Inventory - Revised (DUSI-R). Los análisis descriptivos indicaron que la mayoría de los adolescentes habrían consumido marihuana (84\%) y alcohol (54\%). Respecto al patrón de consumo, el 52\% fue categorizado como uso abusivo o probable dependencia. En cuanto a los problemas asociados con el consumo, los participantes tuvieron una densidad media/superior a la población normativa en los diez dominios investigados por DUSI-R, con énfasis en los Trastornos ( $M=42)$, la Escuela $(M=50)$ y Parejas $(M=55)$. Cabe destacar que el $37 \%$ de la muestra se declaró abstencionista y que las desviaciones de las densidades del problema sugieren una alta heterogeneidad en los niveles de necesidades/dificultades, lo que implica la superación de discursos que promueven la uniformidad de esta población. Palabras clave: alcohol; adolescentes en conflicto con la ley; delincuencia juvenil; consumo de sustancias.
\end{abstract}

\section{Introdução}

A adolescência é, em alguma medida, demarcada pela intensificação de processos maturacionais - corporais e neuropsicológicos. Tais processos, ao mesmo tempo que suscitam novas capacidades, relacionadas a uma melhor adaptação ante as exigências da vida social, também geram vulnerabilidade, pois fomentam comportamentos de busca por autonomia que, por vezes, implicam envolvimento em atividades de risco à saúde física e mental (Kaufmann et al., 2017; Lee et al., 2014). Duas dessas atividades são o uso de substâncias psicoativas e a prática de delitos. Estas remetem a comportamentos que, em geral, iniciam-se na adolescência 
e podem se estender à vida adulta (Moffitt, 2018; Schulenberg, Maslowsky, \& Jager, 2018). Para além dos riscos decorrentes dos efeitos diretos das drogas, o problema/dificuldade com consumo de substâncias também dispara ou agrava outras condições de saúde mental que dificultam o rompimento com a trajetória desviante (Basto-Pereira \& Maia, 2018; Underwood \& Washington, 2016).

Levantamentos mostram que a prevalência de uso de substâncias psicoativas em meio a adolescentes infratores é maior que na população com a mesma faixa etária (Carlini et al., 2010; CNJ, 2012). Mostram também que eles tendem a experimentar drogas e a utilizá-las com regularidade mais precocemente (Salas-Wright, Vaughn, \& González, 2016). No contexto brasileiro, um estudo realizado na extinta Fundação Estadual do Bem-Estar do Menor (FEBEM) de Porto Alegre - RS (Ferigolo et al., 2004) identificou que as substâncias mais utilizadas eram álcool (81\%), tabaco (77\%) e maconha $(69 \%)$, e que a idade média em que teriam sido utilizadas pela primeira era inferior aos 13 anos. O estudo também comparou adolescentes institucionalizados por medida socioeducativa com adolescentes institucionalizados por medida protetiva. Verificou-se que a proporção dos internados por atos infracionais que faziam uso de substâncias era significativamente maior para todas as drogas investigadas, com exceção do tabaco. Em outro estudo realizado na FEBEM, nos municípios de Ribeirão Preto e Sertãozinho, no Estado de São Paulo, Martins e Pillon (2008) encontraram que as substâncias mais utilizadas eram o álcool (97\%), a maconha $(96 \%)$ e o tabaco $(90 \%)$. Adicionalmente, as autoras identificaram correlações significativas entre a idade de início do uso da substância e a idade de cometimento do primeiro delito.

O levantamento nacional mais recente foi realizado pelo Conselho Nacional de Justiça (CNJ, 2012), que contou com a participação de quase a totalidade de adolescentes em conflito com a lei, internados em 320 instituições de todas as regiões brasileiras. Segundo a pesquisa, 75\% dos adolescentes relataram fazer uso de substâncias ilícitas, sendo a maconha $(67 \%)$, a cocaína $(32 \%)$ e o crack $(16 \%)$ as substâncias investigadas mais utilizadas. A prevalência do uso de substância nessa população é preocupante, principalmente quando se compara a adolescentes não judicializados. Em estudo recente com 568 adolescentes em cidade no interior do estado de São Paulo, Galinari, Vicari e Bazon (2019) identificaram que o risco de um adolescente judicializado consumir maconha semanalmente é vinte vezes maior em relação a um adolescente não judicializado.
No fluxo dessas evidências, formou-se a ideia de uma estreita relação entre os dois comportamentos, quando não a de que constituem uma questão unívoca, em que remeteriam a uma só problemática. Essa ideia perpassa a opinião pública e, em alguma medida, o discurso institucional, ampliando o espaço para concepções reducionistas das necessidades/dificuldades dos adolescentes, o que pode contaminar a formulação de políticas públicas na área. Em termos científicos, há consenso de que esses comportamentos possuem alguma relação entre si, mas as evidências indicam ao menos três formas distintas em que o uso de substâncias e o envolvimento em atividades infracionais poderiam estar relacionados: (1) o uso de drogas leva à prática de delitos, (2) a prática de delitos leva ao uso de drogas e (3) o uso de drogas e a prática de delitos compartilham de fatores de risco comuns (Brochu, Brunelle, Plourd, \& Da Silva, 2018; Komatsu, Estevão, \& Bazon, 2018; Leidenfrost, Leonard, \& Antonius, 2017).

Para além da proposição de que, em alguns casos, a droga causa o crime, o que significaria que o uso de substâncias pode influenciar no envolvimento em delitos, concebe-se também que o crime leva ao uso de drogas, uma vez que a prática reiterada de atos infracionais concorre para uma imersão em subculturas nas quais o uso de drogas ilícitas costuma ser aceito e incentivado. Komatsu et al., 2018b encontraram evidências que suportam a existência dessas duas trajetórias em adolescentes de diferentes cidades brasileiras, mas destacam que ambas as perspectivas, de natureza unidirecional, são pouco robustas para explicar a heterogeneidade de trajetórias de uso de substâncias e de condutas infracionais na adolescência. Em uma terceira perspectiva, há a proposição de que a relação droga-crime remete a comportamentos que representam uma orientação desviante geral, resultado da exposição do indivíduo a um conjunto de fatores que são risco para ambas as problemáticas, concomitantemente. Essa perspectiva realça a importância de conhecer as dimensões e a intensidade dos riscos aos quais esses adolescentes estão expostos, pois o início e a continuidade de ambas as condutas desviantes estariam em função desses fatores (Komatsu et al., 2018b; Le Blanc \& Bouthillier, 2003).

Contudo, na literatura nacional, poucos estudos foram encontrados sobre os padrões de uso de substâncias e as dimensões dos riscos aos quais os adolescentes em conflito com a lei estão expostos. Andrade, Silva e Assumpção Jr. (2004) estudaram 116 adolescentes infratores em regime de internação 
e verificaram que $63 \%$ deles faziam uso abusivo de drogas, sendo que a maioria destes apresentava comorbidades psiquiátricas, como transtorno de conduta $(77 \%)$, transtorno depressivo $(60 \%)$, transtorno desafiador opositivo (57\%), transtorno de déficit de atenção e hiperatividade (57\%) e transtorno de ansiedade (57\%). Teixeira, Campos e Martins (2017) identificaram em uma amostra de adolescentes infratores que $87 \%$ faziam uso excessivo de álcool, 93\% podiam ser considerados dependentes de maconha e $73 \%$ faziam uso importante de cocaína. No entanto, a amostra da pesquisa foi constituída por apenas 15 adolescentes infratores, em semiliberdade, o que limita as generalizações possíveis. Com uma amostra de 40 adolescentes em conflito com a lei, Pereira et al. (2018) encontraram que $15 \%$ já haviam experimentado as sensações de fissura e $27 \%$ o sentimento de impotência frente ao uso. $\mathrm{O}$ estudo ainda encontrou outros efeitos negativos decorrentes do uso de substância, como mudanças repentinas de humor (relatado por $47 \%$ ), envolvimento em acidentes de carro (60\%) e dificuldade de se lembrar do que fez $(65 \%)$.

A literatura científica traz amplo suporte para a ideia de que o uso de substância consiste em uma problemática recorrente na vida dos adolescentes em conflito com a lei. É, contudo, fundamental conhecer os problemas associados a ela para entender melhor a relação droga-crime na vida desse segmento específico e para fornecer subsídios à construção de políticas públicas mais contingentes às suas necessidades/dificuldades. Assim, o presente estudo teve como objetivo caracterizar uma amostra de adolescentes em conflito com a lei em relação ao uso de substâncias psicoativas e à incidência de problemas sociais, relacionais e de saúde física e mental que estariam associados ao uso das substâncias. Os objetivos específicos foram: 1) estimar a prevalência do uso de sete categorias de substâncias (álcool, tabaco, maconha, inalantes, alucinógenos, clubdrugs e cocaína/crack) e a idade em que essas substâncias são experimentadas pela primeira vez; 2) estimar a frequência em que essas substâncias são utilizadas; 3) a partir da informação da frequência em que fazem uso de cada substância, classificar os adolescentes em relação ao padrão de consumo (uso experimental, abusivo ou dependência); 4) estimar a intensidade de problemas em dez domínios da vida dos adolescentes: problemas relacionados ao próprio uso de substâncias, comportamento, saúde, saúde mental/transtornos psiquiátricos, sociabilidade/competência social, família, escola, trabalho, relacionamento com pares e lazer.

\section{Método}

\section{Participantes}

Participaram do estudo 120 adolescentes de 13 a 18 anos incompletos $(M=16 ; D P=1,1)$, todos do sexo masculino que, devido ao cometimento de atos infracionais, encontravam-se custodiados na Unidade de Atendimento Inicial da Fundação Centro de Atendimento Socioeducativo ao Adolescente (Fundação CASA), localizada no Núcleo de Atendimento Integrado (NAI) de Ribeirão Preto - SP, aguardando decisão judicial. A maioria se disse solteiro (90\%), sem trabalho $(71 \%)$ e que havia abandonado os estudos $(73 \%)$. A escolaridade dos participantes seguiu a seguinte distribuição: quinto ano do ensino fundamental $(6 \%)$, sexto ano $(12 \%)$, sétimo ano (13\%), oitavo ano $(18 \%)$, nono ano $(25 \%)$, primeiro ano do ensino médio $(13 \%)$, segundo $(6 \%)$ e terceiro $(4 \%)$ ano do ensino médio. Do total, $85 \%$ revelaram não professar qualquer tipo de religião.

Mais da metade da amostra investigada (56\%) tinha histórico de delitos anteriores, tendo passagem pela delegacia de polícia ao menos outra vez. Um número expressivo dos jovens provinha de famílias desconstituídas (83\%), vivendo com uma renda variando entre $\mathrm{R} \$ 500,00$ e $\mathrm{R} \$ 1.500,00$ (89\%), o que as situaria, hoje, nas classes E, D e C2, segundo os parâmentros do Critério de Classificação Econômica Brasil (Associação Brasileira de Empresas de Pesquisa - ABEP, 2018). Destaca-se o fato, segundo relato dos participantes, de um pouco menos da metade (42\%) conviver, em família, com pessoas com passagem pela polícia devido ao envolvimento em atividade ilícita e/ou apresentar o comportamento de uso de substâncias.

\section{Instrumentos}

Dois instrumentos foram utilizados. O primeiro foi uma entrevista estruturada sobre informações sociodemográficas e institucionais, preparada especificamente para a presente pesquisa. As perguntas eram relativas à família dos adolescentes (constituição, renda familiar, presença de familiares com problemas com a justiça e/ou com drogas) e ao próprio jovem (idade, estado civil, religião, trabalho e escolaridade, número de passagens anteriores pelo sistema de justiça). As informações obtidas pela entrevista foram utilizadas unicamente para caracterizar os adolescentes.

O outro instrumento foi o Drug Use Screening Inventory - Revised (DUSI-R). Desenvolvido por Ralph Tarter (1990) para uma avaliação multidimensional do 
comportamento de uso de substâncias e de problemas associados (observando múltiplas áreas da vida do indivíduo avaliado). Trata-se de um questionário composto de 149 questões de autorrelato, cujas respostas "sim" ou "não" possibilitam quantificar a intensidade de problemas em 10 domínios/áreas da vida do adolescente: (1) A do próprio comportamento de uso de substâncias - (1a) frequência do uso e (1b) grau de envolvimento com as drogas e potencial uso abusivo; (2) Problemas de comportamento (isolamento social, expressão da raiva e autocontrole); (3) Saúde (acidentes, ferimentos e doenças); (4) Saúde mental/transtornos (ansiedade, depressão, antissocialidade e sintomas psicóticos); (5) Sociabilidade/ competências sociais (interações sociais, habilidades sociais e assertividade); (6) Família (disfunção, conflito e supervisão parental); (7) Escola (desempenho acadêmico e ajustamento na escola); (8) Trabalho (competência no trabalho e motivação); (9) Relacionamento com os pares (rede social, envolvimento em gangues e qualidade das relações); e (10) Lazer (qualidade das atividades no tempo livre). A gravidade de problemas em um determinado domínio se dá pela maior quantidade de respostas afirmativas.

De Micheli e Formigoni (2000; 2002) realizaram os procedimentos de adaptação e de validação do DUSI-R para a população de adolescentes brasileiros. As análises indicaram forte consistência interna, com o alfa de Cronbach médio entre as 10 escalas de 0,96 (DP $=0,02)$ e coeficiente de fidedignidade pelo método das metades de 0,86 (DP $=0,08)$ e boa acurácia, classificando corretamente $80 \%$ de adolescentes dependentes de drogas e 90\% de adolescentes não dependentes (De Micheli \& Formigoni, 2002).

\section{Procedimentos de Coleta e de Análise de Dados}

O projeto foi aprovado pelo Comitê de Ética em Pesquisa com Seres Humanos da Instituição à qual a pesquisa está vinculada (CAAE 19457313.4.0000.5407). Conforme o mencionado, todos os adolescentes foram recrutados na Unidade de Atendimento Inicial (UAI). A UAI recebe os adolescentes levados à Delegacia Especializada da Infância e Juventude (também situada no NAI da cidade), após apreensão policial. Considera-se que a UAI é porta de entrada do Sistema de Justiça Juvenil. Assim, constitui-se em local privilegiado para o recrutamento de participantes para pesquisas sobre adolescentes em conflito com a lei que partam da premissa da existência de diferenças em padrões comportamentais intragrupo. A UAI concentra em seu espaço adolescentes implicados em um rol variado de delitos, desde os "leves" até os considerados mais "graves".

A coleta de dados foi realizada em uma "Sala de Atendimento Individual", apropriado à entrevista em termos de privacidade. Nesse momento, deixou-se claro ao adolescente que tanto a pesquisa quanto o pesquisador não tinham vínculos com a polícia/com a justiça, garantindo o anonimato das informações. Nas entrevistas, com duração média de 25 minutos, o entrevistador lia a questão ao adolescente, que poderia acompanhá-la em uma cópia que lhe era fornecida, e anotava a resposta do adolescente em uma folha de registro. A aplicação dos instrumentos seguiu esta ordem: questionário sociodemográfico e, em seguida, DUSI-R.

Os dados foram transferidos para planilhas digitais e submetidos a análises estatísticas descritivas. Foi realizado o cálculo das porcentagens de adolescentes que referiram uso de cada uma das substâncias investigadas (prevalência de uso na vida e durante o último mês) e a média e o desvio padrão da idade em que fizeram uso pela primeira vez.

Em seguida, foi construída uma tabela cruzada com o número de adolescentes que relataram uso durante o último mês e as seguintes categorias de frequência de uso: (1) de uma a duas vezes; (2) de três a nove; (3) de 10 a 20; (4) acima de 20 vezes. Essas categorias de agrupamento de respostas são iguais às utilizadas por De Micheli e Formigoni (2004) em seu estudo com adolescentes brasileiros de escolas públicas.

Os dados da frequência de uso durante o último mês foram utilizados para classificar os adolescentes em relação ao padrão de uso de substâncias, segundo as categorias propostas por De Michelli e Formigoni (2000): "abstêmios" (ausência de respostas afirmativas), "uso experimental" (de 1 a 2 respostas afirmativas), "uso abusivo" (de 3 a 7 respostas afirmativas) e "provável dependência" (acima de 7 respostas afirmativas). Foram calculadas as porcentagens de adolescentes em cada uma das categorias mencionadas. Por fim, foram calculadas as médias das densidades de problemas (número de respostas afirmativas dividido pelo número de questões, multiplicado por 100) em cada uma das 10 áreas investigadas.

\section{Resultados}

Sublinha-se, primeiramente, que 12 adolescentes $(10 \%$ da amostra) relataram nunca ter usado qualquer uma das substâncias. A Tabela 1 apresenta, então, os 
resultados descritivos concernentes às frequências absoluta e relativa dos 108 adolescentes que referiram uso de ao menos uma das substâncias, ao longo da vida e durante o último mês que precedeu a coleta de dados, e também apresenta a média de idade em que elas teriam sido utilizadas pela primeira vez.

Destaca-se que 23 adolescentes, ou seja, 21\% dessa subamostra, relataram não ter feito uso de qualquer substância no último mês. Em relação ao uso combinado, destaca-se o binômio maconha-álcool, cujo uso, ao longo do último mês, foi referido por $41 \%$ da amostra. Os adolescentes que referiram uso de alguma substância durante o último mês $(n=108)$ foram contabilizados nas categorias de frequência explicitadas acima, conforme mostra a Tabela 2 .

A partir do padrão de uso revelado pelos adolescentes $(n=120)$, estes foram agrupados em uma das quatro categorias relativas ao padrão atual de uso de substâncias psicoativas de acordo com a seção I(b) do DUSI-R, variando de abstêmios (33\%) até provável dependência (15\%), conforme mostra a Tabela 3.

Nota-se que, considerando os abstêmios e os que fizeram uso experimental, quase metade dos adolescentes não apresentam problemas específicos ao consumo de substâncias, embora possam apresentar problemas em outras áreas em decorrência do uso, mesmo que experimental. A Tabela 4 mostra as médias das densidades de problemas associados ao uso de substâncias nas dez áreas/domínios investigados pelo DUSI-R na amostra total $(n=120)$.

As médias das densidades de problemas variaram de 22,2, no domínio Trabalho, a 55,1, no domínio Relacionamentos com os pares. Observa-se que toda pontuação positiva é indicativa de algum nível de problema.

\section{Discussão}

Este estudo objetivou caracterizar o comportamento de uso de substâncias psicoativas de adolescentes em conflito com a lei, considerando a importância de melhor compreender a relação droga-crime, especialmente na adolescência, levando em conta o imperativo de refletir sobre os conteúdos das intervenções de prevenção e de tratamento (no quadro das medidas socioeducativas), nesse campo. Assim, destaca-se, primeiramente, que, embora o uso de drogas tenha se mostrado bastante prevalente em meio aos jovens investigados, $10 \%$ referiram jamais ter feito uso de qualquer substância, o que, por si, já implica ponderações sobre uma visão simplista de que os comportamentos de uso de substâncias e o de prática de delitos remetam a um só problema. Sabe-se que o uso de substâncias é mais prevalente em meio a adolescentes infratores do que em meio a adolescentes da população (Carline, et al., 2010; CNJ, 2012). No entanto, Komatsu et al., 2018b ressaltam que, entre adolescentes não judicializados, apenas a minoria dos que usam drogas se envolve em delitos. Os autores ponderam que há diferentes relações entre droga e crime e mostram evidências que demonstram as duas atividades - uso de drogas e prática delituosa - compartilham um conjunto de fatores de risco, sendo esses suscetíveis de, cumulativamente, desencadear uma ou as duas problemáticas concomitantemente, a depender de outras circunstâncias/variáveis. Assim, supõem-se que os adolescentes em conflito com a lei estejam, em

Tabela 1.

Porcentagem de Adolescentes que Referiram Uso na Vida e no Último Mês das Substâncias Investigadas

\begin{tabular}{lccc}
\hline & $\begin{array}{c}\text { Uso na vida } \\
(n=108)\end{array}$ & $\begin{array}{c}\text { Uso no último mês } \\
(n=85)\end{array}$ & $\begin{array}{c}\text { Idade que fez uso } \\
\text { pela } 1^{\text {a }} \text { vez }\end{array}$ \\
\hline Substância & $n(\%)$ & $n(\%)$ & Média $(D P)$ \\
maconha & $101(84 \%)$ & $85(71 \%)$ & $12,5(2,0)$ \\
álcool & $68(57 \%)$ & $60(50 \%)$ & $13,7(2,1)$ \\
tabaco & $33(28 \%)$ & $27(23 \%)$ & $12,3(3,4)$ \\
inalantes/solventes & $23(19 \%)$ & $8(7 \%)$ & $13,2(2,4)$ \\
club drugs & $22(18 \%)$ & $9(8 \%)$ & $13,0(2,0)$ \\
alucinógenos & $21(18 \%)$ & $3(3 \%)$ & $*$ \\
cocaína/crack & $17(14 \%)$ & $10(8 \%)$ & $13,4(2,0)$ \\
\hline
\end{tabular}

Nota.* Não há informações sobre a idade em que os alucinógenos teriam sido usados pela primeira vez.

Psico-USF, Bragança Paulista, v. 26, n. 2, p. 229-240, abr./jun. 2021 
Tabela 2.

Porcentagem de Adolescentes que Referiram Uso de cada Substância no Último Mês

\begin{tabular}{lcccc}
\hline Substância & 1 a 2 vezes & 3 a 9 vezes & 10 a 20 vezes & Mais de 20 vezes \\
\hline maconha & $4(3 \%)$ & $6(5 \%)$ & $2(2 \%)$ & $73(61 \%)$ \\
álcool & $2(2 \%)$ & $54(45 \%)$ & $3(3 \%)$ & $1(1 \%)$ \\
tabaco & $0 \%$ & $2(2 \%)$ & $1(1 \%)$ & $24(20 \%)$ \\
inalantes/solventes & $4(3 \%)$ & $1(1 \%)$ & $2(2 \%)$ & $1(1 \%)$ \\
club drugs & $1(1 \%)$ & $7(6 \%)$ & $0 \%$ & $1(1 \%)$ \\
alucinógenos & $1(1 \%)$ & $2(2 \%)$ & $0 \%$ & $0 \%$ \\
cocaína/crack & $2(2 \%)$ & $2(2 \%)$ & $2(2 \%)$ & $4(3 \%)$ \\
\hline
\end{tabular}

Tabela 3.

Prevalência de Adolescentes por Categoria de Padrão de Uso de Substâncias

\begin{tabular}{lcc}
\hline Padrão de uso & $n$ & $\%$ \\
\hline Abstêmios & 40 & $33 \%$ \\
Uso experimental & 18 & $15 \%$ \\
Uso abusivo & 44 & $37 \%$ \\
Provável dependência & 18 & $15 \%$ \\
\hline
\end{tabular}

Tabela 4.

Medidas Descritivas das Densidades de Problemas Associados ao Uso de Substâncias na Amostra Total

\begin{tabular}{lccc}
\hline Domínios & Mediana & Média $(D P)$ & IC $95 \%$ \\
\hline 1. Comportamento de uso & 20,0 & $23,1(24,4)$ & {$[18,2--27,1]$} \\
2. Problemas de comportamento & 32,5 & $35,1(17,2)$ & {$[31,5-37,7]$} \\
3. Saúde & 30,0 & $36(19,3)$ & {$[31,6--38,7]$} \\
4. Transtornos & 40,0 & $41,9(18,1)$ & {$[37,8--44,6]$} \\
5. Sociabilidade/competência social & 28,6 & $34(17,2)$ & {$[30,2--36,7]$} \\
6. Família & 28,6 & $32,1(21,4)$ & {$[27,8--35,7]$} \\
7. Escola & 50,0 & $50(19,4)$ & {$[45,6--52,6]$} \\
8. Trabalho & 20,0 & $22,2(17,3)$ & {$[19,4-25,8]$} \\
9. Relacionamento com pares & 57,1 & $55,1(19,8)$ & {$[50,8-58,2]$} \\
10. Lazer & 50,0 & $45,9(21,6)$ & {$[41,8--49,9]$} \\
\hline
\end{tabular}

Nota. IC = intervalo de confiança.

termos gerais, mais expostos a esse conjunto de fatores de risco que os adolescentes da população.

Em meio aos adolescentes que relataram uso de alguma substância, destaca-se a preferência pelo uso de maconha. Essa substância seria a mais prevalente, tanto em termos de uso na vida (84\%), quanto em termos de uso no último mês (76\%). A prevalência de uso na vida foi maior do que os 67\% encontrados pelo CNJ (2012), em levantamento feito em instituições de internação de todo o Brasil, e que os 11\% encontrados em adolescentes escolares da rede pública e de mesma faixa etária (Carlini et al., 2010). A prevalência de uso no último 
mês foi bastante superior aos 7,2\% encontrados pela Pesquisa Nacional de Saúde do Estudante (PeNSE; IBGE, 2016, p. 91) em amostra de adolescentes de 16 a 17 anos de escolas públicas e privadas.

A preferência pela maconha entre os adolescentes em conflito com a lei pode ser em razão da acessibilidade que esses jovens têm à substância, talvez, devido ao fato de também estarem, em alguma medida, ligados ao tráfico de drogas. Outra explicação pode remeter a duas ordens de motivação (Komatsu et al., 2018b). A primeira refere-se à influência de modelos no meio criminalizado, no qual muitos adolescentes em conflito com a lei estão inseridos, considerando a existência de uma subcultura desviante que fomenta e reforça atividades ilícitas de diferentes tipos. Nessa subcultura, o uso de determinadas drogas é "normal". Assim, muitos adolescentes experimentam e consomem maconha como parte de um processo de socialização. A segunda ordem de motivos remete à busca dos efeitos que a maconha produz, considerando suas propriedades ansiolíticas. Nesse sentido, muitos indivíduos utilizam maconha para reduzir sintomas de estresse e/ou de depressão, uma espécie de uso terapêutico da substância como tentativa de amenizar a ansiedade, a tensão ou o sofrimento que podem decorrer de suas condições de vida. Alguns adolescentes em conflito com a lei podem encaixar-se nesse perfil.

Grande parte dos adolescentes investigados também referiu o uso de álcool (57\% na vida e 50\% no último mês). Apesar de alta, a proporção é sensivelmente menor que a aquela encontrada no VI Levantamento Nacional sobre Consumo de Drogas Psicotrópicas (Carlini et al., 2010), em meio a adolescentes de escolas públicas $(82 \%)$, e ainda menor que a encontrada em meio a adolescentes de escolas privadas $(89 \%)$. A alta prevalência do uso do álcool na população adolescente denota uma alta acessibilidade a essa substância e sugere a existência de aspectos culturais relacionados à motivação para o seu amplo uso no Brasil (Pechansky, Szobot, \& Scivoletto, 2004). Dentro disso, os adolescentes em conflito com a lei não se destacariam, ao contrário do que era esperado (Salas-Wright et al., 2016). O tabaco seria a terceira droga mais frequentemente consumida pelos adolescentes investigados. Um terço (33\%) teria feito uso ao longo da vida e um pouco menos (27\%), ao longo do último mês, proporções próximas aos $31 \%$ encontrado no levantamento nacional com escolares. Nesse sentido, parece não haver preferência especial por essa substância no segmento dos adolescentes em geral.
Menos prevalentes, mas ainda assim referidas por alguns participantes deste estudo, aparecem os inalantes/solventes (19\% na vida; 7\% no último mês), as club drugs (18\% na vida; $8 \%$ no último mês), os alucinógenos (18\% na vida; $3 \%$ no último mês) e a cocaína/ crack (14\% na vida; $8 \%$ no último mês). As proporções contrastam com os resultados do VI Levantamento Nacional sobre Consumo de Drogas Psicotrópicas, no qual as prevalências encontradas foram de $12 \%$ (rede pública) e de $15 \%$ (rede privada) para os inalantes; $0,3 \%$ para a metanfetamina; $2,5 \%$ para a anfetamina; $4 \%$ para o êxtase (substâncias consideradas club drugs); 1\% para o LSD (uma substância alucinógena); 5\% para cocaína; $1 \%$ para crack. Embora as prevalências, em comparação com as das drogas anteriores, possam ser consideradas baixas, elas são maiores em meio aos adolescentes em conflito com a lei. Observa-se, contudo, que há uma diferença relevante entre os números de "experimentadores" (uso na vida) e de "consumidores" (uso no último mês). Apesar dos riscos inerentes às alterações imediatas que as substâncias acima podem produzir no organismo, o uso experimental não necessariamente representa um problema (NIDA, 2014). Nese sentido, o dado sobre cocaína/ crack, especialmente, contradiz o senso comum, pelo qual, normalmente, entende-se que muitos infratores são dependentes desse tipo de substância.

O uso problemático de todas as substâncias remete prioritariamente a um padrão de uso frequente (que não pode ser considerado experimental), em razão dos riscos que se assume com relação aos efeitos negativos das substâncias a médio e a longo prazo (Hasin et al., 2013). Nessa ótica, os resultados obtidos denotam que, apesar da maioria dos adolescentes investigados tenha experimentado diversas substâncias, é apenas em relação a algumas delas que uma porcentagem considerável apresentaria uso problemático. Novamente, as três substâncias que mais se destacaram em termos de prevalência também foram as que mais se destacaram em termos de frequência de uso: maconha, álcool e tabaco. Em relação à maconha, $73 \%$ dos adolescentes referiram tê-la utilizado mais de 20 vezes ao longo do último mês. No estudo de De Micheli e Formigoni (2004) junto a 6 mil adolescentes de escolas públicas, apenas 1,5\% dos adolescentes referiu uso superior a 20 vezes. Apesar de a maconha ser comumente classificada como uma droga "leve", seu uso durante a adolescência é bastante preocupante. $\mathrm{O}$ consumo elevado pode resultar em alterações neuroanatômicas, reduzindo o volume de regiões pré-frontais do cérebro (Lubman, Cheetham, 
\& Yucel, 2015). Funcionalmente, estudos transversais e longitudinais apontam que o uso crônico de maconha está associado a déficits de atenção, de aprendizagem, de memória, de velocidade de processamento de informação e a um declínio neuropsicológico acentuado (Jacobus \& Tapert, 2014; Meier et al., 2012; Meruelo, Castro, Cota, \& Tapert, 2017). Déficits nessas funções concorrem para dificuldades de autorregulação, que consistem em um importante fator de risco para diversos desfechos negativos, incluindo a prática de delitos (Komatsu, Costa \& Bazon, 2018; Moffitt, 2018).

Em segundo lugar, destaca-se o álcool, utilizado de três a nove vezes ao longo do último mês por $45 \%$ dos participantes. Assim como a maconha, o uso frequente de álcool pode produzir alterações anatômicas e funcionais no cérebro, sobretudo em um organismo em desenvolvimento (Meruelo et al., 2017). O álcool está igualmente associado a prejuízos nas funções executivas de atenção e de memória de trabalho, atinentes ao autocontrole. Os possíveis efeitos adversos são ainda mais preocupantes se considerarmos que, em muitos casos, o uso das substâncias seria, além de frequente, crônico e estável no tempo. Nesse sentido, é importante ressaltar que a idade média de início de uso da maconha foi 12,5 anos e a de início de uso de álcool, 13,7 anos. Dessa forma, alguns estariam consumindo essas substâncias há cerca de 3,5 anos e 2,3 anos, respectivamente (considerando a idade média na amostra, de 16 anos). Nesse sentido, há evidências de que quanto mais cedo se inicia o consumo, maior o risco de agravar o padrão de uso, concorrendo também para uma maior prevalência de transtornos relacionados (Moffitt, 2018; Salas-Wright et al., 2016). Ademais, muitos fariam um uso combinado das duas substâncias psicoativas (41\%), o que potencializa seus efeitos e, com isso, os riscos associados a curto, médio e longo prazo (NIDA, 2014).

A terceira substância de uso mais frequente foi o tabaco: $20 \%$ dos adolescentes referiram tê-lo consumido mais de 20 vezes ao longo do último mês. Num primeiro plano, mediante uso tão reiterado de tabaco, pontua-se sobre os riscos à saúde física desses jovens: doenças respiratórias, câncer, artrite e diabetes mellitus (Harvey, Chadi \& Canadian Paediatric Society, Adolescent Health Committee, 2016). Além de constituírem-se problemas em si, essas condições patológicas podem ter impacto na saúde mental do indivíduo e de sua família. Em um segundo plano, embora os efeitos imediatos da nicotina no comportamento do usuário sejam menos perceptíveis, se comparados às outras substâncias investigadas, já se dispõem de evidências de que a nicotina também provoca em adolescentes (embora não em adultos) prejuízos nas funções executivas de atenção e de autocontrole, sendo eles, por isso, mais vulneráveis aos efeitos dessa droga (Counotte et al., 2011; Goriounova \& Mansvelder, 2012; Harvey \& Chadi, 2016). Yuan, Cross, Loughlin e Leslie (2015) ainda argumentam que a exposição à nicotina, a longo prazo, sensibiliza o cérebro para outras drogas, aumentando o risco de abuso de outras substâncias subsequentemente, o que agravaria a problemática. De fato, em estudo nacional recente, Komatsu, Costa, Galinari, Carpio, Bazon (2020) identificaram que poliusuários de substâncias possuem risco substancialmente maior de problemas de saúde física e mental, além de maior envolvimento em situações de violência.

Considerando que mais da metade dos adolescentes investigados poderiam ser classificados nas categorias de uso abusivo (37\%) ou de provável dependência (15\%), é possível que muitos já estariam experimentando os efeitos negativos do consumo acentuado e crônico dessas substâncias, que intensificam suas dificuldades pessoais, relacionais e sociais, as quais retroalimentam seus comportamentos desviantes. Por conseguinte, os problemas enfrentados por esses jovens, se não pioram, estabilizam-se em condições de vida problemática. Nesse sentido, a densidade de problemas desses adolescentes é substancialmente maior que a média da população normativa obtida por De Micheli e Formigoni (2000; 2002), em todos os domínios. Além dos problemas associados ao próprio uso de substâncias psicoativas e dos problemas de comportamentos, todas as outras oito áreas investigadas são muito importantes para o pleno desenvolvimento humano: saúde, transtornos, sociabilidade/competência social, família, escola, trabalho, pares e lazer. Nesse sentido, sugere-se que jovens com múltiplos problemas necessitem de intervenções multimodais, que suscitem alterações nas várias áreas atinentes a suas problemáticas.

Por fim, há que se sublinhar que um pouco menos da metade dos adolescentes investigados seriam abstêmios $(33 \%)$ ou teriam um padrão de uso de substâncias experimental (15\%). Esses adolescentes estariam em risco de desenvolver um padrão de uso de substâncias mais problemático, seja pelo fato de apresentarem algum envolvimento com a prática de infrações, o que parece suscetível de aumentar a acessibilidade ou motivação de uso a alguns tipos de drogas, seja pelo fato de apresentarem problemas em alguns domínios da vida, para além daqueles que serão gerados em função da apreensão policial, das tensões e dos conflitos que podem emergir 
nos diferentes contextos de vida em razão do estigma social. Todavia, esses adolescentes, de partida, não têm uma necessidade de atenção especializada no quesito "drogadição". A rigor, esse subgrupo não se diferencia significativamente da maioria dos jovens da população em relação às drogas. Assim, tem-se, em realidade, uma amostra de jovens em conflito com a lei heterogênea em suas características - necessidades/dificuldades conforme apontamentos da literatura (McCuish, 2017), o que implica ultrapassar o senso comum e discursos que propalam a uniformidade desse segmento.

\section{Considerações Finais}

O presente estudo buscou caracterizar o padrão de uso de substâncias psicoativas em adolescentes em conflito com a lei. Identificou-se que a maioria desses jovens experimenta muitas substâncias e que, em média, apresentam elevada frequência de consumo de maconha, álcool e tabaco, a ponto de mais da metade poderem ser classificados como tendo um uso abusivo ou uma provável dependência. Todavia, identificou-se que uma proporção também alta, de um pouco menos da metade (48\%), poderia ser considerada abstêmia ou como tendo um padrão de uso experimental. De todo modo, a grande maioria dos adolescentes possuiriam problemas em diversas áreas da vida, acima do que estudos anteriores encontraram em adolescentes da população. Hipotetiza-se que esses fatores podem atuar como desencadeadores e, em seguida, mantenedores dos comportamentos delituosos e de uso de substâncias.

Conquanto, cabe mencionar aqui duas limitações importantes em relação ao alcance e generalização dos resultados e das inferências. A primeira é que consiste em um estudo exploratório e descritivo, o que deve ser levado em conta para a interpretação das informações. A segunda consiste no fato de a amostra ser de conveniência e estar sujeita aos vieses sistêmicos de busca e apreensão dos mecanismos de controle estatais, o que pode ocasionar em uma sub-representação de determinados estratos e super-representação de outros. Por essas razões, salienta-se a necessidade de estudos prospectivos para determinar com clareza a relação entre as variáveis, focalizando a cronologia dos comportamentos delituosos, do de uso das substâncias e os demais problemas nas outras esferas da vida. Igualmente importante, em investigações futuras, será dispor de método de coleta de dados outros que o baseado em autorrelato somente, dada a fragilidade inerente a esse tipo de informação, quando se trata de estudos sobre comportamento humano, bem como recrutar adolescentes em outras regiões do país com características sociais e demográficas distintas daquelas abarcadas pelo presente estudo.

Por último, será muito interessante, em termos teóricos e também de aplicação prática, verificar se padrões determinados de uso substâncias e de problemas nas diferentes áreas se associam a padrões diferentes de implicação em delitos como, por exemplo, maior engajamento ou menor engajamento, maior implicação em delitos contra o patrimônio ou maior implicação em delitos violentos (contra a pessoa). A identificação e a caracterização de subgrupos, se existentes, pode ajudar a pensar de modo ainda mais refinado sobre o tipo de medida socioeducativa pertinente a cada adolescente e sobre os conteúdos dos programas de acompanhamento que necessitam, permitindo focalizar necessidades/dificuldades específicas, em vez de se adotar um plano de tratamento genérico e massificado, ao qual cabe aos adolescentes "se encaixar".

\section{Referências}

Andrade, R., Silva, V., A., \& Assumpção-Junior, F. B. (2004). Preliminary data on the prevalence of psychiatric disorders in Brazilian male and female juvenile delinquents. Brazilian Journal of Medical and Biological Research, 37(8), 1155-1160. doi: https:// doi. org/10.1590/S0100-879X2004000800005)

Associação Brasileira de Empresas de Pesquisa - ABEP. (2018). Alterações na aplicação do Critério Brasil, válidas a partir de 16/04/2018. Recuperado de http://abep.org/Servicos/Download.aspx?id=14

Basto-Pereira, M., \& Maia, Â. (2018). Persistence in crime in young adults with a history of juvenile delinquency: The role of mental health and psychosocial problems. Int J Ment Health Addiction, 16, 496-506. doi: 10.1007/s11469-017-9847-7

Brochu, S., Brunelle, N., Plourde, C., \& Da Silva, J. (2018). Drugs and crime: A complex relationship (3ed). Ottawa: University of Ottawa Press.

Carlini, E. L. A., Noto, A. R., Sanchez, Z. V. D. M., Carlini, C. M. A., Locatelli, D. P., Abeid, L. R., et al. (2010). VI Levantamento Nacional sobre o Consumo de Drogas Psicotrópicas entre Estudantes do Ensino Fundamental e Médio das Redes Pública e Privada de Ensino nas 27 Capitais Brasileiras - 2010. São Paulo: CEBRID 
- Centro Brasileiro de Informações sobre Drogas Psicotrópicas, UNIFESP. Brasília: SENAD - Secretaria Nacional de Políticas sobre Drogas. Recuperado de https://www.cebrid.com.br/ vi-levantamento-estudantes-2010/

CNJ. (2012). Panorama nacional: A execução das medidas socioeducativas de internação. Brasília: Conselho $\mathrm{Na}$ cional de Justiça. Recuperado de https://www.cnj. jus.br/wp-content/uploads/2011/02/panorama_ nacional_doj_web.pdf

Counotte, D., Goriounova, N., Li, K., Loos, M., van der Schors, R. C., Schetters, D, Schoffelmeer, A. N., Smit, A. B., Mansvelder, H. D., Pattij, T., \& Spijker, S. (2011). Lasting synaptic changes underlie attention deficits caused by nicotine exposure during adolescence. Nature Neuroscience, 14, 417-419. doi: $/ 10.1038 / \mathrm{nn} .2770$

De Micheli, D., \& Formigoni, M. L. O. S. (2000). Screening of drug use in a teenage Brazilian sample using the Drug Use Screening Inventory (DUSI). Addictive Behaviors, 25, 683-691. doi:10.1016/ s0306-4603(00)00065-4

De Micheli, D., \& Formigoni, M. L. (2002). Psychometric properties of the Brazilian version of the drug use screening inventory. Alcoholism, Clinical and Experimental Research, 26(10), 1523-1528. doi: 10.1097/01.ALC.0000033124.61068.A7

De Micheli, D., \& Formigoni, M. L. (2004). Drug use by Brazilian students: Associations with family, psychosocial, health, demographic and behavioral characteristics. Addiction, 99(5), 570-578. doi: 10.1111/j.1360-0443.2003.00671.x

Ferigolo, M., Barbosa, F. S., Arbo, Elisangela., Malysz, A. S., Stein, A. T., \& Barros, H. M. T. (2004). Prevalência do consumo de drogas na FEBEM, Porto Alegre. Revista Brasileira de Psiquiatria, 26(1), 10-16. doi: 10.1590/S1516-44462004000100006

Galinari, L. S., Vicari, I. D. A., \& Bazon, M. R. (2019). Fatores associados ao cometimento de atos infracionais na adolescência. Psico (Porto Alegre), 50(4), e-34094. doi: 10.15448/1980-8623.2019.4.34094

Goriounova, N. A., \& Mansvelder, H. D. (2012). Shortand long-term consequences of nicotine exposure during adolescence for prefrontal cortex neuronal network function. Cold Spring Harbor Perspectives in Medicine, 2(12). doi: 10.1101/cshperspect.a012120
Harvey, J., Chadi, N., \& Canadian Paediatric Society, Adolescent Health Committee. (2016). Preventing smoking in children and adolescents: Recommendations for practice and policy. Pediatrics \& Child Health, 21(4), 209-214. doi: 10.1093/pch/21.4.209

Hasin, D. S., O’Brien, C. P., Auriacombe, M., Borges, G., Bucholz, K., Budney, A., ... Grant, B. F. (2013). DSM-5 Criteria for substance use disorders: recommendations and rationale. The American Journal of Psychiatry, 170(8), 834-851. doi: 10.1176/appi. ajp.2013.12060782

Instituto Brasileiro de Geografia e Estatística - IBGE. (2016). Pesquisa nacional de saúde escolar. Rio de Janeiro: Instituto Brasileiro de Geografia e Estatística. Recuperado de https://biblioteca.ibge.gov.br/visualizacao/livros/liv97870.pdf

Jacobus, J., \& Tapert, S. F. (2014). Effects of cannabis on the adolescent brain. Current Pharmacentical Design, 20(13), 2186-2193. doi: /10.2174/13816128113199990426

Kaufmann, T., Alnæs, D., Doan, N. T., Brandt, C. L., Andreassen, O. A., \& Westlye, L. T. (2017). Delayed stabilization and individualization in connectome development are related to psychiatric disorders. Nature Neuroscience, 20(4), 513-515. doi: 10.1038/ nn. 4511

Komatsu, A. V., Costa, R. C. S., \& Bazon, M. R. (2018). Delinquência juvenil: Relações entre desenvolvimento, funções executivas e comportamento social na adolescência. Rev. Bras. Polít. Públicas, 8(2), 979999. doi: 10.5102/rbpp.v8i2.5289

Komatsu, A. V., Costa, R. C. S., Galinari, L. S., Carpio, R., Bazon, M. R. (2020). Substance use and involvement in situations of violence: A typological study of a Brazilian population-based sample. International Annals of Criminology, 57(1), 1-23. doi:10.1017/ cri. 2020.3

Komatsu, A. V., Estevão, R., \& Bazon, M. R. (2018b). Relações entre drogas e crime: modelos teóricos e pesquisas empíricas com adolescentes no Grupo de Estudos e Pesquisa em Desenvolvimento e Intervenção Psicossocial da Universidade de São Paulo. Em C. Agra \& M. Gomes (Eds.), Criminologia Integrativa. Belo Horizonte: D’Placido.

Le Blanc, M., \& Bouthillier, C. (2003). A developmental test of the general deviance syndrome with adjudicated girls and boys using hierarchical confirmatory 
factor analysis. Criminal Behaviour and Mental Health, 13(2), 81-105. doi:10.1002/cbm.533

Lee, F. S., Heimer, H., Giedd, J. N., Lein, E. S., Šestan, N., Weinberger, D. R., \& Casey, B. J. (2014). Adolescent mental health - opportunity and obligation: Emerging neuroscience offers hope for treatments. Science, 346(6209), 547-549. doi: 10.1126/ science. 1260497

Leidenfrost, C. M., Leonard K. E., \& Antonius, D. (2017). Alcohol, drugs, and crime. Em V. Van Hasselt \& M. Bourke (Eds.), Handbook of Behavioral Criminology. Springer.

Lubman, D. I., Cheetham, A., \& Yucel, M. (2015). Cannabis and adolescent brain development. Pharmacology \& Therapentics, 148, 1-16. doi: 10.1016/j. pharmthera.2014.11.009

Martins, M. C., \& Pillon, S. C. (2008). A relação entre a iniciação do uso de drogas e o primeiro ato infracional entre os adolescentes em conflito com a lei. Caderno de Saúde Pública, 24(5):1112-1120. doi: 10.1590/S0102-311X2008000500018

McCuish, E. C. (2017). Substance Use Profiles Among Juvenile Offenders: A Lifestyles Theoretical Perspective. Journal of Drug Issues, 47(3), 448-466. https://doi.org/10.1177/0022042617699197)

Meier, M. H., Caspi, A., Ambler, A., Harrington, H., Houts, R., Keefe, R. S., ... Moffitt, T. (2012). Persistent cannabis users show neuropsychological decline from childhood to midlife. Proceedings of the National Academy of Science, 109(40), E2657-E2664. doi: 10.1073/pnas.1206820109

Meruelo, A., Castro, N., Cota, C., \& Tapert, S. (2017). Cannabis and alcohol use, and the developing brain. Behavioural Brain Research, 325, 44-50. doi: 10.1016/j.bbr.2017.02.025

Moffitt, T. (2018). Male antisocial behaviour in adolescence and beyond. Nature Human Behaviour, 2(3), 177-186. doi: 10.1038/s41562-018-0309-4

NIDA. (2014). Drugs, Brains, and Behavior: The science of addiction. Recuperado de https://d14rmgtrwzf5a. cloudfront.net/sites/default/files/soa.pdf

Pechansky, F., Szobot, C. M., \& Scivoletto, S. (2004). Uso de álcool entre adolescentes: Conceitos, características epidemiológicas e fatores etiopatogênicos. Brazilian Journal of Psychiatry, 26(1), 14-17. doi: 10.1590/S1516-44462004000500005

Pereira, C. C. M., Zambalde, C. G. S., Lambert, C. C., Costa, P. M., Machado, J. S. A., Botti, N. C. L. (2018). Características pessoais e familiares entre adolescentes infratores. Rev. enferm. Cent.-Oeste Min; 6(2), 2212-2222. doi:10.19175/recom.v6i2.991

Salas-Wright C. P., Vaughn M. G., \& González J. M. R. (2016). Adolescent drug abuse and delinquency. Em C. Salas-Wright, M. Vaughn \& J. Reingle, Drug Abuse and Antisocial Behavior (pp. 129-155). New York: Palgrave Macmillan. doi: 10.1057/978-1-137-55817-6_6

Schulenberg, J., Maslowsky, J., \& Jager, J. (2018). Substance use and abuse during adolescence and the transition to adulthood are developmental phenomena: Conceptual and empirical considerations. Em H. E. Fitzgerald \& L. I. Puttler (Eds.), Alcohol use disorders: A developmental science approach to etiology (pp. 199-222). Oxônia: Oxford University Press.

Tarter, R. (1990). Evaluation and treatment of adolescent substance abuse: A decision tree method. American Journal of Drug and Alcohol Abuse, 16(1-2), 1-46. https://doi.org/10.3109/00952999009001570)

Teixeira, P. S., Campos, T. E., \& Martins, R. A. (2017). Consumo de álcool e outras drogas em adolescentes infratores de uma unidade de semiliberdade do interior de estado de São Paulo. Colloquium Humanarum, 14(4), p.15-20. doi:10.5747/ch.2017.v14. n4.h329

Underwood, L. A., \& Washington, A. (2016). Mental illness and juvenile offenders. International Journal of Environmental Research and Public Health, 13(2), 228. doi: 10.3390/ijerph13020228

Yuan, M., Cross, S. J., Loughlin, S. E., \& Leslie, F. M. (2015). Nicotine and the adolescent brain. The Journal of Physiology, 593(16), 3397-3412. doi:10.1113/ JP270492

Recebido em: 13/08/2019

Reformulado em: 15/06/2020

Aprovado em: 10/08/2020 
About the authors:

André Vilela Komatsu é Professor contratado do Departamento de Sociologia da Faculdade de Filosofia, Letras e Ciências Humanas da Universidade de São Paulo e pesquisador do Núcleo de Estudos da Violência (NEV-USP). Membro do Grupo de Estudos e Pesquisa em Desenvolvimento e Intervenção Psicossocial (GEPDIP). Psicólogo, Mestre em Ciências (área: Psicologia) e Doutor em Psicologia (área: Saúde e Desenvolvimento), todos pela Universidade de São Paulo, com estágio doutoral na Universitat de Barcelona.

ORCID: https://orcid.org/0000-0001-8508-6787

E-mail:avk@usp.br

Elvio Luciano Bono é Psicólogo clínico do Hospital das Clínicas de Ribeirão Preto. Docente do curso de Psicologia no Centro Universitário UNIFAFIBE. Mestre em Ciências pela Faculdade de Filosofia, Ciências e Letras de Ribeirão Preto da Universidade de São Paulo (FFCLRP-USP).

ORCID: https://orcid.org/0000-0003-3682-4899

E-mail: elviobono@yahoo.com.br

Marina Rezende Bazon é Professora Associada do Departamento de Psicologia da Faculdade de Filosofia, Ciências e Letras de Ribeirão Preto da Universidade São Paulo (FFCLRP-USP). Atua como docente na Graduação e na Pós-Graduação da FFCLRP-USP e coordena o Grupo de Estudos e Pesquisa em Desenvolvimento e Intervenção Psicossocial (GEPDIP). Mestrado em Ciências pela Universidade Montreal/Canadá e Doutorado em Psicologia pela Universidade de São Paulo.

ORCID: https://orcid.org/0000-0002-8037-8710

E-mail:mbazon@fffclrp.usp.br

\section{Contact:}

Departamento de Psicologia

Faculdade de Filosofia, Ciências e Letras de Ribeirão Preto

Universidade de São Paulo

Av. Bandeirantes, 3900

Ribeirão Preto-SP, Brasil

CEP: 14040-901 